\title{
Overt Oculomotor Behavior Reveals Covert Temporal Predictions
}

\author{
Alessandro Tavano ${ }^{1 *}$ and Sonja A. Kotz ${ }^{2,3}$ \\ ${ }^{1}$ Department of Neuroscience, Max Planck Institute for Empirical Aesthetics, Frankfurt am Main, Germany, ${ }^{2}$ Department of \\ Neuropsychology and Psychopharmacology, Faculty of Psychology and Neuroscience, Maastricht University, Maastricht, \\ Netherlands, ${ }^{3}$ Department of Neuropsychology, Max Planck Institute for Human Cognitive and Brain Sciences, Leipzig, \\ Germany
}

Our eyes move in response to stimulus statistics, reacting to surprising events, and adapting to predictable ones. Cortical and subcortical pathways contribute to generating context-specific eye-movement dynamics, and oculomotor dysfunction is recognized as one the early clinical markers of Parkinson's disease (PD). We asked if covert computations of environmental statistics generating temporal expectations for a potential target are registered by eye movements, and if so, assuming that temporal expectations rely on motor system efficiency, whether they are impaired in PD. We used a repeating tone sequence, which generates a hazard rate distribution of target probability, and analyzed the distribution of blinks when participants were waiting for the target, but the

OPEN ACCESS

Edited by:

Adonis Moschovakis, University of Crete, Greece

Reviewed by:

Daniel Thomas Smith, Durham University, United Kingdom Shlomit Yuval-Greenberg, Tel Aviv University, Israel

*Correspondence: Alessandro Tavano alessandro.tavano@ae.mpg.de; alessandro.tavano.office@gmail.com

Specialty section:

This article was submitted to Cognitive Neuroscience,

a section of the journal

Frontiers in Human Neuroscience

Received: 13 August 2021

Accepted: 14 January 2022

Published: 11 February 2022

Citation:

Tavano A and Kotz SA (2022) Overt Oculomotor Behavior Reveals Covert Temporal Predictions.

Front. Hum. Neurosci. 16:758138. doi: 10.3389/fnhum.2022.758138 target did not appear. Results show that, although PD participants tend to produce fewer and less temporally organized blink events relative to healthy controls, in both groups blinks became more suppressed with increasing target probability, leading to a hazard rate of oculomotor inhibition effects. The covert generation of temporal predictions may reflect a key feature of cognitive resilience in Parkinson's Disease.

Keywords: predictions, temporal expectations, eye movements, Parkinson's disease, hazard rate

\section{INTRODUCTION}

Blinks are defined as the temporary closure $(\approx 0.3 \mathrm{~s})$ of both eyes via rapid movements of both the upper and lower lids: the closing and closed phases of the movement are extremely rapid $(<0.1 \mathrm{~s})$, while the opening phase is slower $(\approx 0.2 \mathrm{~s}$, Kwon et al., 2013). On average, healthy human adults blink every 3-5 s $\approx$ 12-20 blinks per minute, Fatt and Weissman, 1992). Blinks help preserve the integrity of the ocular surface (lubrication, shielding from light and dirt, relieving eye muscle fatigue, Hall, 1945). However, blink frequency far exceeds such basic physiological needs, and there is evidence that arousal and attention drive blink frequency to change depending on whether at any given moment sensory information processing can be chunked (Wascher et al., 2015), when a release of attention from external stimulation is required (Nakano et al., 2013), or when fulfilled expectations indicate the end of cognitive processing (Ichikawa and Ohira, 2004).

In general, spontaneous blink rates decrease when attention is directed to incoming, external stimuli, particularly during experimental trials (Van Opstal et al., 2016) and when sustained, continuous attention is required to successfully complete a task (Maffei and Angrilli, 2018). It is unclear whether the component of attention that modulates blinking probability is strictly under dopaminergic control (Maffei and Angrilli, 2018; Sescousse et al., 2018), but there is evidence that Parkinson's patients with dyskinetic symptoms often exhibit increased eye movement rates 
(Karson, 1983), possibly as a consequence of intracortical dishibition (Stinear and Byblow, 2003; Ammann et al., 2020). Indeed, eye movement disorders may present one of the early symptoms of Parkinson's Disease onset (Jung and Kim, 2019). However, while the blinking rate is likely a confounded measure as it could be due to either attentional demands or fatigue (Maffei and Angrilli, 2018), the temporal distribution of blink movements or blink timing appears to be a reliable and unconfounded index of participants' engagement in a task (Ichikawa and Ohira, 2004; Nakano and Miyazaki, 2019).

Temporal attention is reflected in the hazard rate distribution, which normalizes true stimulus probability using the survival function, that is, the probability that the event has not yet occurred (Luce, 1986). Recent work showed that blinks and saccadic movements are suppressed (oculomotor inhibition) before the onset of predictable targets (Abeles et al., 2020). For uniformly distributed stimulus onset times, the perceived probability of target onset is assumed to monotonically increase as time elapses. It follows that blink probability at each target position should diminish with increasing temporal expectations. As cortical beta disorganization in Parkinson's disease has been associated with reduced sensitivity to temporal regularities (te Woerd et al., 2015), and the generation of temporal expectations has been linked to motor cortical activity (Morillon and Baillet, 2017), we tested the distribution of blinks in Parkinson's patients (PD) and a healthy control group (HC) matched for gender, age, and cognitive performance. All participants completed an auditory task which required detecting the onset of a target sound in a continuous attention mode. Auditory stimulation sequences were composed of the continuous repetition of four standard tones followed by a fifth non-target, deviant tone. All sounds were delivered using a fixed stimulus onset asynchrony interval (isochronous stimulation). Target sounds occurred rarely (20\% of sequences) and unpredictably (randomized distribution) within the repeating sequence, equiprobably substituting a standard tone in either position 2, 3, or 4 , hence giving rise to the hazard rate of response times (see stimulus structure section). We hypothesized that if the orienting of attention in time giving rise to expectations depends on the functional integrity of motor cortical, then the distribution of blinks in time in PD and HC should differentially reflect the temporal statistics of target onset. Specifically, we expected PD patients to be less efficient than HC in suppressing blinks with the increasing probability of target onset as attention moved from position 2 to position 4 within each sound sequence.

\section{MATERIALS AND METHODS}

\section{Participants}

The experiment was conducted at the Max Planck Institute for Human and Cognitive Brain Sciences in Leipzig (Germany). Sixteen participants diagnosed with Parkinson's Disease (PD, 9 males, 7 females) were selected (mean age $=63.9$ years, $\mathrm{SD}=$ 6.8). Sixteen healthy adult individuals (Healthy controls, HC), matched in age (mean $=63.9$ years, $\mathrm{SD}=7.1$ ) and gender, were also recruited from the Institute's database. Education level was also matched $(\mathrm{PD}$, mean $=5.6$ years, $\mathrm{SD}=1.2 ; \mathrm{HC}$, mean $=5.7$ years, $\mathrm{SD}=1.3)$. HC participants self-reported no neurological or psychiatric disorders or therapies involving the central nervous system. All participants signed a written informed consent complying with the Declaration of Helsinki on human experimentation. The study was approved by the Ethics Committee of the University of Leipzig, Germany.

\section{Neuropsychological Profile}

The two experimental groups were also cognitively matched on a battery of neuropsychological tests (see Table 1, reporting means and standard deviations within parenthesis): Mini Mental Test (Tombaugh and McIntyre, 1992); Tower of London (Shallice, 1982); Trail Making Test A and B (Tombaugh, 2004); Working memory-Digit Span Forward, maximal $\mathrm{N}$ of numbers recalled [Wechsler, 1997, Backward, maximal $\mathrm{N}$ of numbers recalled (Wechsler, 1997). For all pairwise comparisons, all ts ${ }_{(30)} \leq-0.73$, all ps $\geq 0.465]$.

\section{Clinical Profile}

The average illness duration in participants with PD was 3.78 years ( $S D=2.63)$, with only two participants having been diagnosed for more than 6 years (15 and 11 years). Most patients (11 out of 16) presented with both tremors and akinetic rigidity, while 3 presented solely with akinesia and 2 with tremors. The average Höhn and Yahr index (Höhn and Yahr, 1967) was 2.03 ( $\mathrm{SD}=0.53$, range $1-3)$, suggestive of bilateral involvement preserved balance functions. Asymmetry in body symptoms was equally distributed (right side $=8$ ). On the UPDRS motor scale (Goetz et al., 2007), the mean was 13.5 $(\mathrm{SD}=5$, range $7-21)$, indicative of minimal to mild slowness and movement abnormality. All participants with $\mathrm{PD}$ were pharmacologically treated, predominantly with Levodopa and Ergot-dopamine agonists.

\section{Stimulus Structure}

Stimuli were three 50 -ms pure tones ( $5 \mathrm{~ms}$ rise/fall), organized into continuously repeating five-tone sequences, binaurally presented via loudspeakers at $80 \mathrm{~dB}$ SPL and generated using Matlab (version 7, Mathworks, Natick, MA). The five-tone sequence was composed of four standard tones followed by a non-target deviant tone (Figure 1A). Standards were 440 $\mathrm{Hz}$ in pitch (A4 on the equal tempered scale, presented 900 times, $75 \%$ global stimulus probability), non-target deviants were $494 \mathrm{~Hz}$ (B4, presented 240 times, 20\% global stimulus probability). A rare target $(349 \mathrm{~Hz}, \mathrm{~F} 4$, presented 60 times, $20 \%$ of sequences, $5 \%$ global stimulus probability) occurred equiprobably $(1 / 3)$ at one among standard positions 2,3 , or 4. To detect a target, participants had to attentively listen to each incoming sequence, whether it contained a target or not; within each sequence, internal target probability was predicted to changed with elapsed time, generating a hazard rate distribution (Figure 1B, upper panel). Denoting the survival probability ("the event has not yet occurred") as $1-F(t)$, where $F(t)$ is the cumulative distribution function, the hazard function is then: $\mathrm{h}(\mathrm{t})$ $=\mathrm{f}(\mathrm{t}) /(1-\mathrm{F}(\mathrm{t}))$. There was a maximum of one target per sequence, and minimally two successive sequences without targets before the next target-containing sequence. Stimulus sequences were 
TABLE 1 | Neuropsychological results.

\begin{tabular}{lcccr}
\hline Group & Mini Mental State & Tower of London & Trail Making Test A and B & Digit Span Fw \\
\hline HC & $29.12(0.85)$ & $16.18(1.42)$ & A: $40.56(12.06)$, B: $77.62(25.32)$ & $6.50(1.00)$ \\
PD & $28.87(0.99)$ & $15.43(2.06)$ & A: $39.37(12.21)$, B: $82.37(32.49)$ & $6.68(1.04)$
\end{tabular}

A

Experimental design

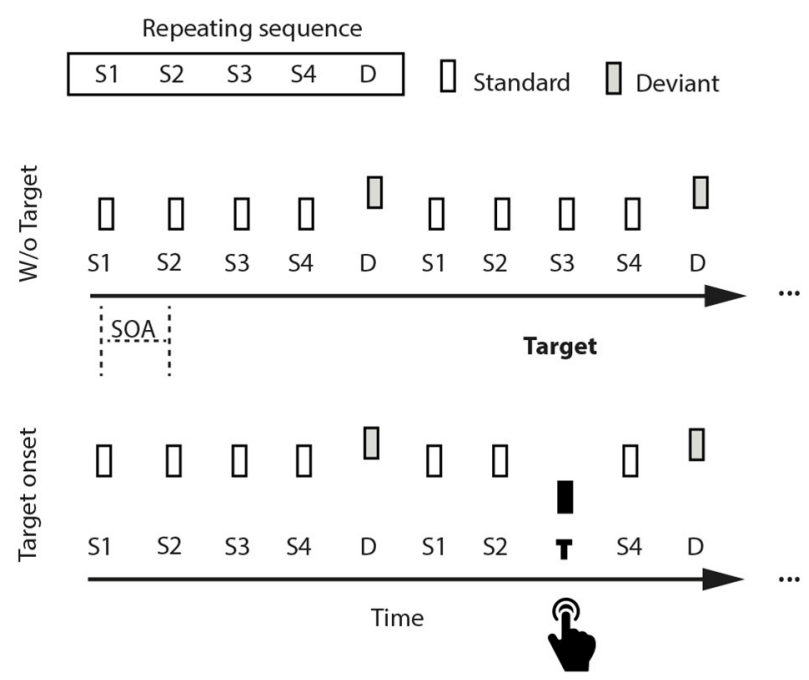

\section{Target distribution}
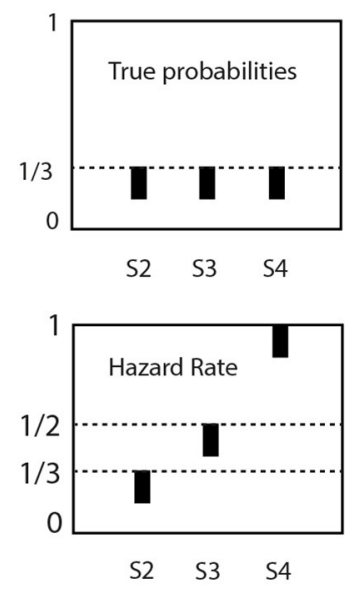

FIGURE 1 | Experimental design: (A) Pure tones were isochronously distributed in continuously repeating five-tone sequences, composed of four standard tones (440 $\mathrm{Hz}$ ) and a final non-target deviant tone $(494 \mathrm{~Hz})$. Target tones were lower in pitch $(349 \mathrm{~Hz})$, and appeared in $20 \%$ of the sequences $(=5 \%$ of the tones). (B) Tones would appear equiprobably either in position two, three, or four within a sequence (upper left panel). Potential rare target position is used as a proxy for elapsed time. The hazard rate distribution increases target probability with target position (upper right panel).

delivered using Presentation $\odot$ software (version 12.0, www. neurobs.com) running on a Windows PC.

\section{Experimental Design}

Participants sat in an electrically shielded, sound-attenuated chamber, and fixated a white cross on a black computer screen at a distance of 1 meter while listening to the auditory sequences. They responded to target tone onset by pressing a button on an external response box, using their preferred hand. Participants were unaware of target distribution, and were instructed to respond to the onset of target tones as accurately and fast as possible by pressing a button on a response box. They trained in a short block of 60 experimental randomly distributed tone sequences containing three targets. The training phase was repeated maximally once. If errors were made (Missing, False Alarm), the training block was repeated until no errors were detected. Experimental tone sequences were delivered with a constant 750-ms stimulus onset asynchrony (SOA), corresponding to a $1.34 \mathrm{~Hz}$ stimulus rate (three 5-min blocks).

\section{EEG Recording}

Electroencephalographic (EEG) data were collected using a 26 scalp Ag/AgCl electrode set (BrainAmp, 10-20 system): Fp1, Fpz, Fp2, F7, F3, Fz, F4, F8, FT7, FC3, FC4, FT8, T7, C3, Cz,
C4, T8, CP5, CP6, P7, P3, Pz, P4, P8, O1, O2. Two external electrodes were placed at right and left mastoid sites, and four additional electrodes were placed at both eye canthi (leftLateral, rightLateral), and above and below the right eye (lowerVertical, upperVertical) to record eye movements (electrooculogram, EOG). An online reference was placed on the left mastoid and the sternum served as ground. Electrode impedance was kept below $5 \mathrm{KOhm}$. EEG/EOG sampling rate was set to 500 $\mathrm{Hz}$, with online high-pass filtering at $0.01 \mathrm{~Hz}$. The resulting continuous recordings were visually inspected and pruned from non-stereotypical artifacts or extreme voltage changes values. An Independent Component Analysis (ICA, Infomax algorithm, Bell and Sejnowski, 1995, as implemented in the EEGLAB toolbox, Delorme and Makeig, 2004) was performed on pruned, offline highpass-filtered at $1 \mathrm{~Hz}$ and lowpass-filtered at $45 \mathrm{~Hz}$ (Kaiser window, Beta 5.6533, filter order points 9056 and 184, transition bandwidth 0.2 and $10 \mathrm{~Hz}$, respectively), standardized (z-score) continuous data. Using the SASICA toolbox for EEGLAB (Chaumon et al., 2015), ICs reflecting blinks/vertical eye movements and lateral eye movements were identified by a correlation threshold of 0.7 with bipolarized vertical and lateral EOG channels. The SASICA toolbox also identified ICs likely to reflect muscle artifacts, using autocorrelation (lag = $20 \mathrm{~s}$ ), as well as those reflecting bad electrodes via a measure of focal 
topography (threshold at 7 standard deviations relative to the mean across electrodes). The ICA results were then copied back to the pruned, standardized original continuous EEG data highpass-filtered at $0.1 \mathrm{~Hz}$ (lowpass $45 \mathrm{~Hz}$ ). Eye-movementrelated ICs, both vertical/blink-related and horizontal, ranged between 2 and 5 per participant, with at least a vertical/blinkrelated component per participant.

\section{Blink Modeling}

Blinks were individually modeled using the best signal selected out of a subset comprising the vertical EOG channel (both lowerV and upperV), a subset of frontal electrodes (in our case: Fp1, Fp2, Fz, F3, F4) and frontally focused independent components (ICs) representing blinks or vertical eye movements according to the Blinker toolbox pipeline (https://github.com/ VisLab/EEG-Blinks; Kleifges et al., 2017). The Blinker algorithm first bandpasses the signal $(1-20 \mathrm{~Hz})$, then determines the intervals with an $\mathrm{SD}>1.5$ standard deviations above the signal mean (min interval $=50 \mathrm{~ms}$, min separation between intervals $=50 \mathrm{~ms}$ ). A fitting process follows by first finding specific landmarks for each blink interval, such as the maximal value within the interval, and the zero crossings immediately to the left and right of each max value, and then computing for each potential blink the best linear fits for the inner $80 \%$ of the upstroke and down-stroke, respectively. The $\mathrm{R}^{2}$ of left and right fit lines with the actual blink trajectory measures how close the potential blink is to a stereotypical blink. Then, the blinkamplitude ratio (BAR) is computed by dividing the average amplitude of the signal between the blink left and right zero crossings by the average amplitude of the positive portion of the signal comprised between the preceding blink right zero crossing and the current blink left zero crossing, as well as the current blink right zero crossing and the following potential blink right zero crossing (or end of signal if the current blink is the last one). Potential blinks with a BAR outside the range [3-20] are not included in the final computation ("used" signal, see below).

Next, Blinker determines "good" blinks (upStroke and downStroke R2 > 0.90), "better" blinks (upStroke and downStroke R2 > 0.95), and "best" blinks (up-stroke and downstroke R2 > 0.98). To eliminate extraneous eye movements from actual blinks, two further criteria are satisfied: 1) The positive amplitude by velocity ratio $(\mathrm{pAVR}=3)$, calculated from the left zero crossing to the maximal amplitude of each blink, distinguishes between the sharp rise of saccades (large velocity) and the more curved one proper to blinks; 2) The maximum amplitude distribution criterion eliminates blinks with low $\mathrm{R}^{2}$ and with amplitude vastly away from the "best" blink median (Threshold $=5$ robust standard deviations -1.48 times the median absolute deviation from the median-for "best" blinks, 2 for for "good" blinks). The resulting blinks constitute the "used" blinks set, which inform the analysis at an individual participant level (minimum number of blinks to stable estimates $=20$ ).

\section{Analysis of Blink Distributions}

The Blinker pipeline was run on continuous, clean EEG datasets. One participant from the PD group was marked as an outlier as far as blink counts were concerned $(N=556)$ and thus was removed from further analysis, together which the gender- and age-matched HC participant. The final group was thus composed of 30 participants, 15 per group. Then, blink landmarks were copied back to the EEG trial structure, and finally epochs were extracted based on the repeating 5 -tone sequences which did not contain a target $(0-3,500 \mathrm{~ms})$. This approach allowed analyzing the distribution of blinks in time as participants waited for a potential target, without any confounding effect from target onset. For each epoch, we marked the positions in time of blink maximal values (peaks), while the rest of the EEG data were zeroed out, obtaining vectors of blink peak distributions in time.

Participants were first compared for the total number of blinks (counts) and median blink-to-blink interval using a onesided Wilcoxon rank sum test for equal medians, with the assumption that HC would outperform PD participants. The choice of a non-parametric statistical test was motivated by the non-gaussian distribution of blink counts (Kolmogorov-Smirnov test, all ps $<1.645^{\star} 10^{-15}$ ). Blink counts were subject to a robust regression analysis with bisquare weighting of the residuals (Matlab function robustfit.m), to asses the the relationship between $\mathrm{HC}$ and PD blink generators. The effect of age in driving blink counts was also tested, using both robust regression and Spearman correlation.

To assess the degree to which blink timing was sensitive to the auditory stimulus rate $(1.34 \mathrm{~Hz})$, blink epochs were concatenated into a single vector for each participant. A Fast Fourier Transform (FFT) analysis ( $N=8192$ data points, normalized dividing by $\mathrm{N}$ ) was run on a hundred concatenated blink vector per participant in each group. The average peak power differences between HC and PD at sequence rate $(0.267 \mathrm{~Hz})$, stimulus rate $(1.34 \mathrm{~Hz})$, and first harmonic of the stimulus rate $(2.67 \mathrm{~Hz})$ were compared to their group threshold using a Wilcoxon signed rank test, and to each other using a one-sided Wilcoxon rank sum test (effect size $r=\mathrm{Z} / \sqrt{\text { Samplesize }}$ for one sample/paired samples, $r=$ $\mathrm{Z} / \sqrt{\text { Samplesize } 1+\text { Samplesize2 }}$ for independent samples).

Next, we turned to the analysis of median blink distributions within the repeating sequence. First, for each participant blink peak latencies were binned using a 20 -ms bin size. Then, bin counts were normalized by the total number of blinks, and smoothed using a moving median of 5 bins. To obtain a measure of regularity in blink distribution across the repeating sequence, we employed the Nelder-Mead simplex direct search algorithm (Image Analyst, 2021) and optimized the search for the best fit for 5 Gaussian distributions on the median distributions across participants in each group, using a sigma of 20 and taking each tone interval's middle point as an initial guess for the mean or peak of each Gaussian. We then calculated the dissimilarity between HC and PD median histograms using $\chi^{2}$ are a measure of distance: $\operatorname{sum}\left((x i-y i)^{2} /(x i+y i)\right) / 2$. We tested the significance of the distance value using a bootstrapping approach $(1,000$ randomizations). Then, for each participant we collected the value at the each grand median fitted Gaussian peak within each sound interval, and compared them across groups using a series of Wilcoxon rank sum tests, FDR-corrected. Finally, by regressing blink frequency against the positional order of potential Target stimulus onset (positions 2,3 , and 4), we obtained peri-stimulus estimates of hazard rate effects in blink distributions-from -300 
to $+\mathbf{3 0 0} \mathbf{~ m s}$ relative to potential target onset -, which were tested for significance using a one-sample permutation test based on the t-statistic (Groppe et al., 2011; one-sided). Significance was determined for $p=0.05$.

\section{Probabilistic Saccade Estimation}

As a partially independent measures of ocolomotor disorders in participants with $\mathrm{PD}$, we resorted to calculating saccade probability and duration. An impairment in saccadic initiation, leading to a more variable onset of saccadic movements than matched healthy controls, has been shown to characterize patients PD from early on in the disease progression (Terao et al., 2011). Furthermore, saccade intrusions-characterized by involuntary saccades away and back to a fixation point, characterize oculomotor system functioning in PD (White et al., 1983), adding to variance in saccade probability distribution. We selected a probabilistic algorithm which detects saccadesas distinct from peri-blink saccadic movements-using an unsupervised training period (between 50 and $200 \mathrm{~s}$ ), and uses expectation maximization to learn the parameters of Gaussian likelihood distributions for saccades (Toivanen et al., 2015; https://github.com/bwrc/eogert). Two parameters were selected: saccade probability for each detected event, and saccade duration. A Wilcoxon rank sum test was used to detect significant differences in mean variance between PD and $\mathrm{HC}$.

\section{A}

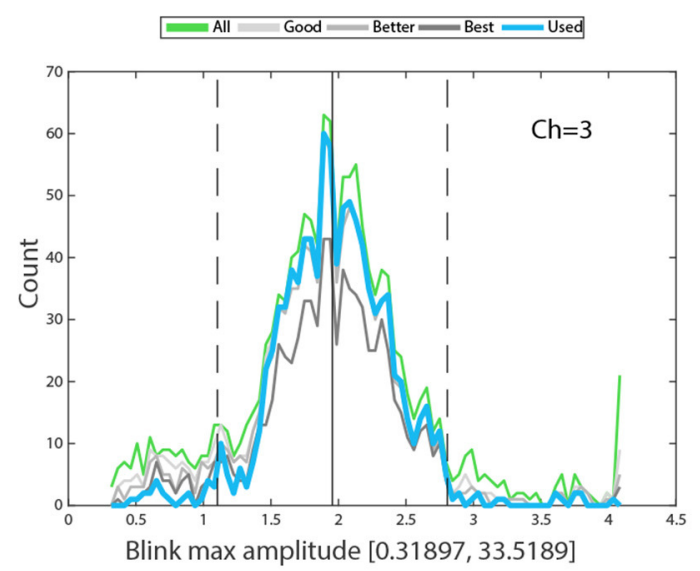

C

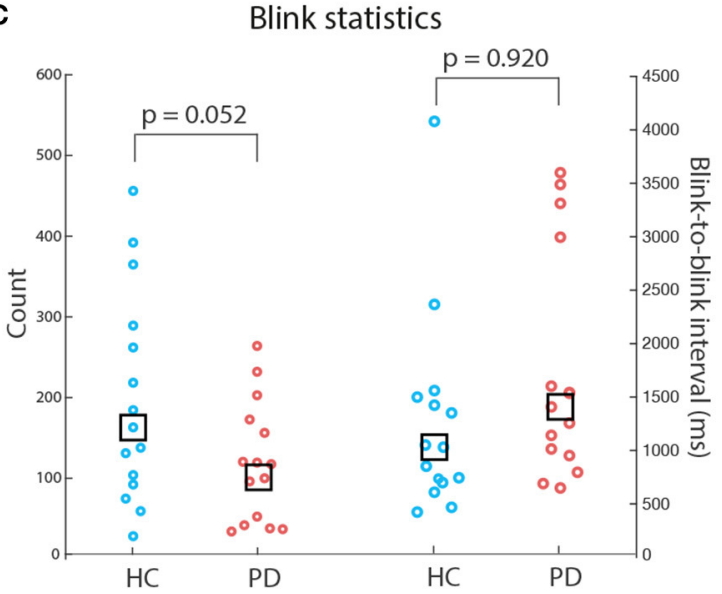

B Blink model PD

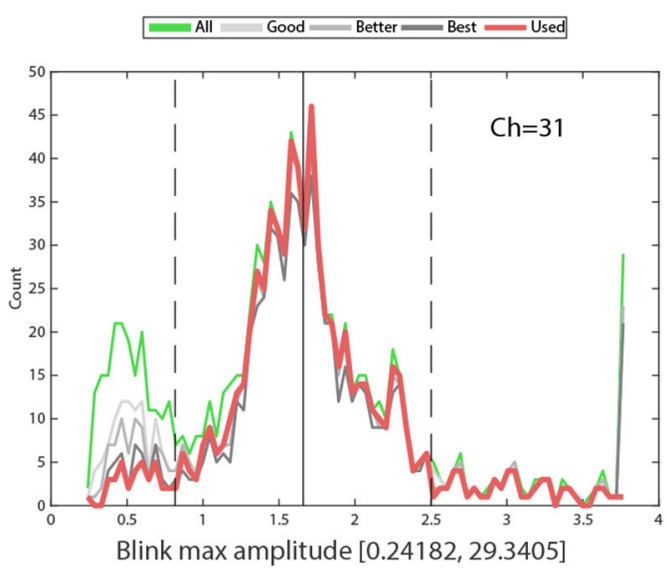

D Blink correlation structure

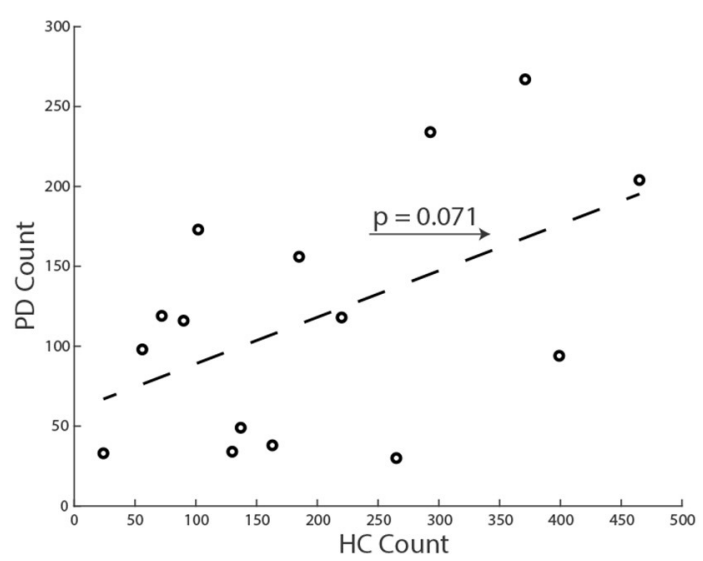

FIGURE 2 | Blink models: (A) Exemplary blink models for participant number 1 of the HC group. The green line depicts the amplitude distribution of all potential blinks: Amplitudes are measured in standard deviations, to avoid the confounding effects of differences in mean blink amplitudes across participants. Blink range on the $x$-axis: Notice that the right tail of the distribution is interrupted because most large amplitude values were outliers. The blue line depicts the blink distribution selected for further analysis. For details, see the Materials and Method section. (B) Exemplary blink models for participant \#1 of the PD group. The red line depicts the blink distribution selected for further analysis. (C) There was a tendency to a significant difference favoring $\mathrm{HC}$ in total blink counts. There was no significant difference between $\mathrm{HC}$ and PD on mean interblink interval. Notice that the median interval is similar across groups. The largest median blink interval for both groups corresponds to physiological intervals. (D) A robust regression fit shows a tendency for matched participants from both groups to perform similarly, hinting at possible underlying common factors driving blink frequency. 


\section{RESULTS}

\section{Blink Models}

Figures 2A,B display illustrative blink modeling results for participant number 1 of both groups. In both cases, the right tail of the distribution contains outliers that are eliminated based on the distance from the best blinks distribution (up-stroke and down-stroke R2 > 0.98). A Wilcoxon rank sum test of the difference between the number of blinks in HC and in PD failed to reach significance: $Z=1.61, p=0.052$, HC median number of blinks $=163$, PD median number of blinks $=116$. We then checked for the physiological realness of the interblink intervals, and found that values for both groups were comparably within expected values: $\mathrm{HC}$ median interblink interval $=1,037$ ms (range: $650-3,638), P D$ median interblink interval $=1,419 \mathrm{~ms}$ (range: 422-4,125), $Z=-1.41, p=0.920$. With the exception of one participant in each group, all medians were below 3,500 ms, likely reflecting the chunking effect of attention to the repeating tone sequence (see Figure 2C). The concentration of individual median values at the lower portion of the range suggests an attractive effect of stimulus rate on blink rate. A robust regression fit between HC blink counts and PD blink counts failed to reach significance $\left[t_{(13)}=1.959, p=0.071\right]$ (see Figure 2D). When we averaged blink counts across groups and regressed the results against age in years, we found no significant fit (Spearman $\rho=$ $-0.215, p=0.503$ ), suggesting that in our samples age did not appear to be driving changes in blink frequencies.

\section{Blink Distribution Reflects Stimulus Structure}

To explore how stimulus structure influenced the temporal distributions of blinks, we concatenated all selected epochs and submitted the resulting vector to a Fast Fourier Transform (FFT) analysis. Using a Wilcoxon signed rank test, we found a significant peak at stimulation frequency $(1.34 \mathrm{~Hz})$ in each group: $\mathrm{HC}, Z=2.89, p=0.002, r=0.74$ (reference power $=3^{\star} 10^{-05}$ ); $\mathrm{PD}, Z=1.98, p=0.047, r=0.51$ (reference power $\left.=2^{\star} 10^{-05}\right)$. However, there was a significant difference in peak power between the groups: HC median $=1.098^{*} 10^{-04}$, $\mathrm{PD}$ median $=4.644^{\star} 10^{-05}, Z=1.825, p=0.034, r=0.33$. There was no significant group peak, nor a group difference at the first harmonic of the stimulus rate $(2.67 \mathrm{~Hz})$ : all ps $\geq 0.079$. Similarly, there were no significant findings at the repeating sequence frequency $(0.266 \mathrm{~Hz})$ : all ps $\geq 0.187$ (see Figure 3).

\section{Blink Rates Encode Temporal Predictions}

The Nelder-Mead algorithm allowed us to optimally fit 5 Gaussians on the median of the median blink distributions for each group. For HC, the number of iterations was 719 , with a mean residual of $6.447^{\star} 10^{-4}$. For $\mathrm{PD}$, the number of iterations was 1228 , with a mean residual of $7.515^{\star} 10^{-4}$ (see Figures 4 A,B, respectively). We measured histogram similarity using $\chi^{2}$ as a distance measure, and found that-globally-the distribution of blinks across the repeating sound sequence did not differ (distance $=1.494, p=0.73$, bootstrapping distribution, 1,000 repetitions). However, when we tested the differences in blink

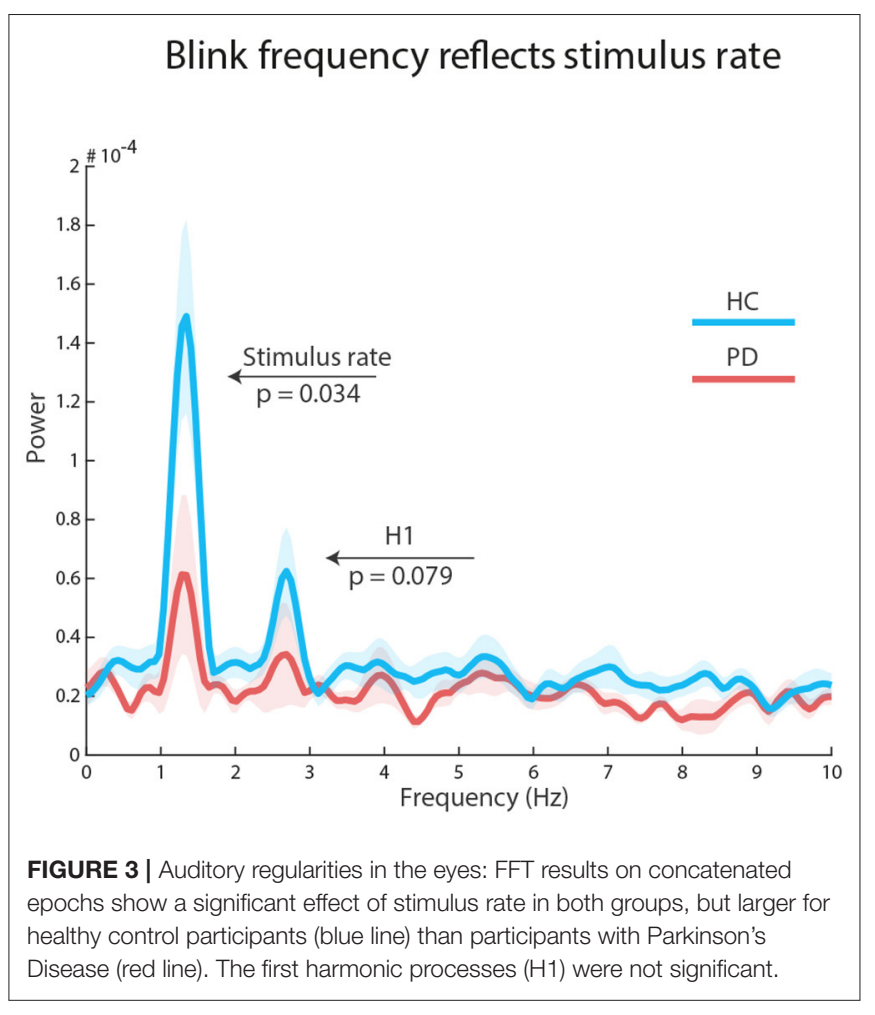

frequency (pristine values, that is before applying the movingaverage smoothing) at fitted curve peak within each sound interval, using the fitted values for $\mathrm{HC}$ as reference also for participants with $\mathrm{PD}$, we then found that the two groups differed in the S3 interval, that is at the center of the sequence (original $p=0.007$, FDR $p=0.01$ ), which corresponds also to the middle point in the attentive searchlight for a potential target onset (Figure 4C).

We then used a robust linear fit approach to calculate, for each peristimulus bin point, the slope of values across the onset of sounds at S2, S3, and S4. For both groups, we found a significant hazard-rate effect on blink frequency in the pre-stimulus period only (Figure 4D): all cluster Ts leq-2.79, all cluster ps leq 0.029; $\mathrm{HC}-220$ to $200 \mathrm{~ms}$, PD $-180 \mathrm{~ms}$; negative slopes indicate the amount of decrease with each potential Target interval. Pre-stimulus oculomotor activity became more inhibited with increasing waiting time across positions S2, S3, and S4.

\section{Saccadic Movements}

The similarity in blink temporal distribution between participants with $\mathrm{PD}$ and HCs becomes more relevant on the background of the significant difference between the two groups in saccadic movements estimated from electrooculographic data. Average saccade probability displyed larger variance in participants with $\mathrm{PD}($ mean $=0.014)$ than in HCs (mean $=$ $0.011): Z=2.13, p=0.032, r=0.54$. However, average saccade duration did not differ: $Z=0.43, p=0.663$. This suggests a disorder in saccade initiation in participants with $\mathrm{PD}$, detectable 
A

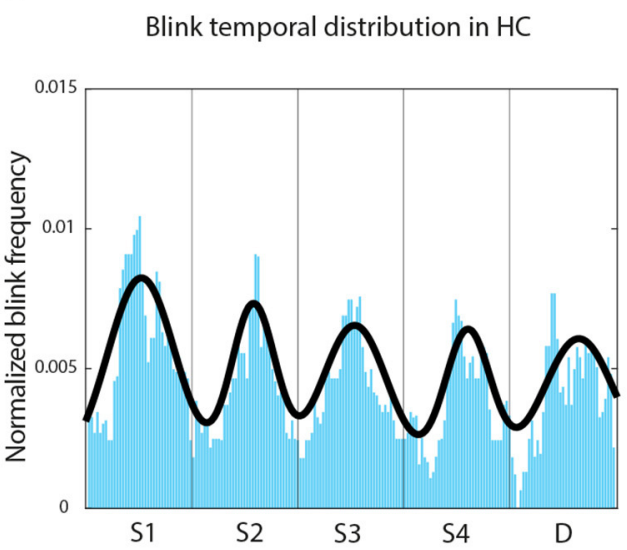

C

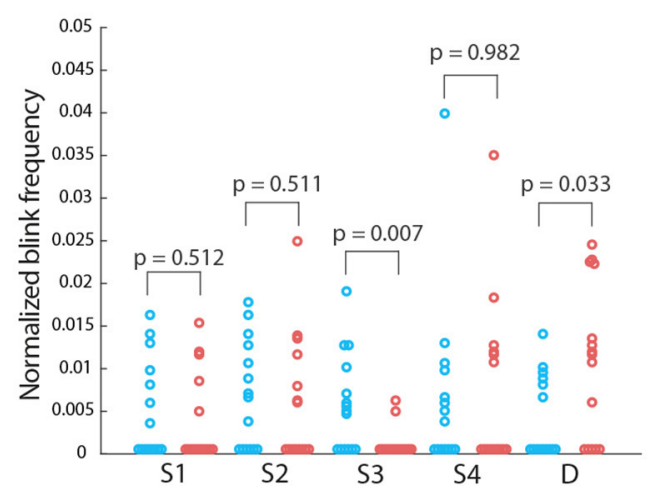

B

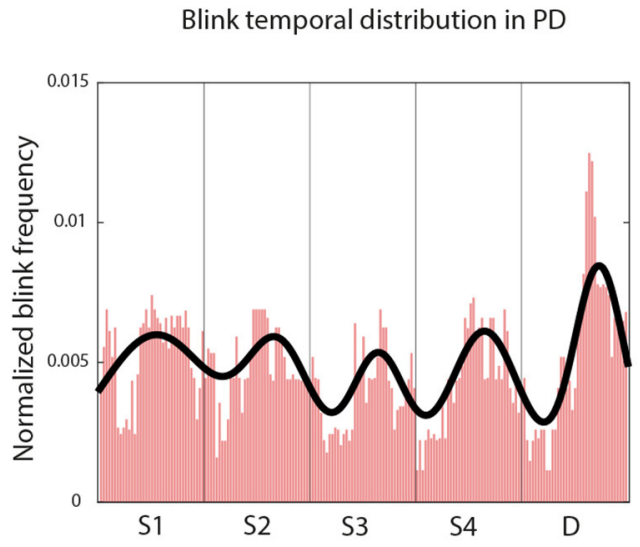

D Peri-stimulus hazard-rate effects

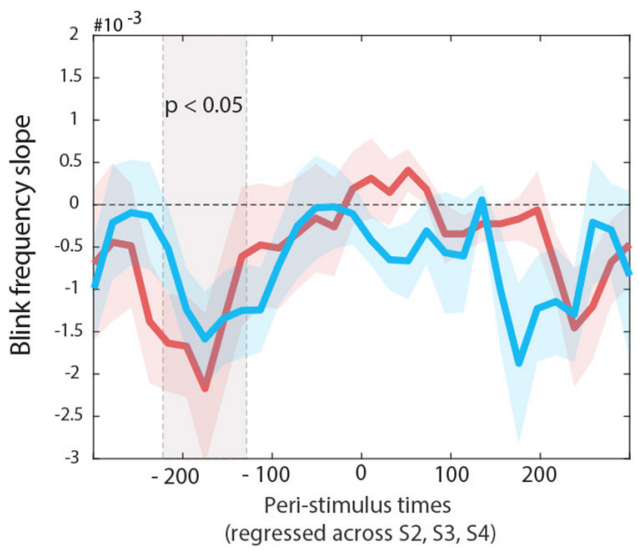

FIGURE 4 | Temporal organization of blink onset: (A) Median of blink frequency medians estimates across time for HCs. The Gaussian fit highlights the regularity in blink peak distribution following entrainment to stimulus regularity. (B) The same approach for PD participants displays a less organized structure. (C) The main difference between the two distribution lies with how blinks reflect the onset of S3. (D) The gray shade indicates mean blink frequency slopes for both HC and PD which display significant oculomotor inhibition effects; colored shaded areas indicate standard error of the mean.

even in the context of isochronous auditory stimulation driving entrainment in blink onset.

\section{DISCUSSION}

When stimulus statistics in the environment drive our attention toward the potential onset of a target event, changes occur at both central and autonomic nervous system levels, thereby modulating all motor effectors, not just those required to press a button. Indeed, recent work suggests that temporal predictions are reflected by eye movements, such as saccades and blinks (Abeles et al., 2020), that are partially under voluntary and partially under involuntary control. When we approach the probable onset time of a target event, ocular movements are suppressed, in order to avoid diverting attention to other stimuli (saccades) or suppressing sensory input (blinks). However, in everyday situations the uncertainty about when a target event will occur adds to the uncertainty about whether a target will occur at all. We tested whether oculomotor inhibition occurs for targets whose chance is globally very low (20\%). Furthermore, by comparing the performance of healthy controls (HC) and genderand age-matched Parkinson's Diseases (PD) patients, we measured the extent to which temporal predictions conveyed via oculomotor inhibition depend on general oculomotor fitness, which is impaired in PD. Overall, PD participants tended to produce less blinks than HCs, but the temporal organization of inter-blink-intervals is similar to that of healthy controls. Previous work showed that, in spontaneous blinking conditions and for a cohort between 40 and 89 years, mean blink amplitude and peak velocity decreased with age, but blink rate as such was not affected (Sun et al., 1997). In our case, age was not a significant factor in determining blink counts, although the effect of age on blink counts might have been overridden by the entraining effects of the stimulation structure. 
We also found that inter-blink-intervals in both groups tend to follow the regular auditory stimulation rate $(750 \mathrm{~ms}$, $1.34 \mathrm{~Hz}$, Figure 3), although $\mathrm{HC}$ outperformed participants with $\mathrm{PD}$. This finding suggests that motor impairment in our sample of participants with PD, including oculomotor saccadic impairments, did not prevent the locking of blink frequency to stimulus statistics. However, oculomotor impairment in PD partially affected the organization of blink peak distribution (Figures 4A-C). Although the sequence-based distance between blink histograms for HC and PD was not significant, we found that in participants with PD blinks were significantly less likely to occur in response to the third sound of each repeating sequence. Previous work showed that spontaneous blinks in PD participants with mild and moderate severity were either abnormally reduced or increased relative to HC (Korosec et al., 2006). PD participants in that study displayed a more advanced motor impairment (UPDRS motor scale score) than in our patient sample, and participants were tested off medication, while the patients in our sample were tested on medication. The lack of an off medication condition is a limit to our findings, as it would have provided a test for blink entrainment. However, our study assesses oculomotor functionality within a continuous attention condition, that is under under stressful attentional demands (Maffei and Angrilli, 2018), suggesting resilience in patients' performance.

When we regressed blink probability across potential Target positions (S2, S3, S4), we found evidence of a hazard rate organization of blink onset probability in both groups. Oculomotor inhibition progressively increased while waiting for a potential target (Figure 4D). Importantly, as our analysis was run on the repeating sequences that did not contain a target, oculomotor inhibition was purely driven by cognitive expectancies for future target onset. The "hazard rate" of oculomotor activity is evident in the prestimulus period only,

\section{REFERENCES}

Abeles, D., Amit, R., Tal-Perry, N., Carrasco, M., and Yuval-Greenberg, S. (2020). Oculomotor inhibition precedes temporally expected auditory targets. Nat. Commun. 11:3524. doi: 10.1038/s41467-020-17158-9

Ammann, C., Dileone, M., Pagge, C., Catanzaro, V., Mata-Marín, D., HernándezFernández, F., et al. (2020). Cortical disinhibition in Parkinson's disease. Brain 143, 3408-3421. doi: 10.1093/brain/awaa274

Bell, A.J., and Sejnowski, T.J. (1995). An information-maximization approach to blind separation and blind deconvolution. Neural Comput. 7, 1129-1159. doi: 10.1162/neco.1995.7.6.1129

Chaumon, M., Bishop, D.V., and Busch, N.A. (2015). A practical guide to the selection of independent components of the electroencephalogram for artifact correction. J. Neurosci. Methods 250, 47-63. doi: 10.1016/j.jneumeth.2015.02.025

Delorme, A., and Makeig, S. (2004). EEGLAB: an open source toolbox for analysis of single-trial EEG dynamics including independent component analysis. J. Neurosci. Methods 134, 9-21. doi: 10.1016/j.jneumeth.2003.10.009

Fatt, I., and Weissman, B.A. (1992). Physiology of the Eye: An Introduction to the Vegetative Functions. Boston, MA: Butterworth-Heinemann; Reed Publishing.

Goetz, C.G., Fahn, S., Martinez-Martin, P., Poewe, W., Sampaio, C., Stebbins, G.T., et al. (2007). Movement disorder society-sponsored revision of the unified Parkinson's disease rating scale (mds-updrs): process, format, and clinimetric testing plan. Mov. Disor. 22, 41-47. doi: 10.1002/mds.21198 consistent with previous findings (Tavano et al., 2019; Abeles et al., 2020). Motor disorganization as a consequence of PDat least in as far as it affects the oculomotor system-did not prevent the computation of evolving target probability in time, which is a key component of the processes generating temporal expectations. This likely preserves in patients a sufficient fit with the environment, whose statistics are inherently time-dependent.

\section{DATA AVAILABILITY STATEMENT}

The raw data supporting the conclusions of this article will be made available by the authors, without undue reservation.

\section{ETHICS STATEMENT}

The studies involving human participants were reviewed and approved by Ethics Committee of the University of Leipzig, Germany. The patients/participants provided their written informed consent to participate in this study.

\section{AUTHOR CONTRIBUTIONS}

AT and SK devised research hypothesis, wrote and revised draft, and approved submission. AT created scripts for data collection and analyzed data. Both authors contributed to the article and approved the submitted version.

\section{ACKNOWLEDGMENTS}

The authors wish to thank Ina Koch for data collection, Jana Kynast for the acquisition of neuropsychological data, and the two reviewers for helpful criticism. Patient data collection was supported by DFG KO 2268/3-1 to SK.
Groppe, D.M., Urbach, T.P., and Kutas, M. (2011). Mass univariate analysis of event-related brain potentials/fields I. A critical tutorial review. Psychophysiology 48, 1711-1725. doi: 10.1111/j.1469-8986.2011.01273.x

Hall, A. (1945). The origin and purposes of blinking. Br. J. Ophthalmol. 29, 445-467.

Höhn, M. M., and Yahr, M. D. (1967). Parkinsonism: onset, progression and mortality. Neurology 17, 427-442. doi: 10.1212/wnl.17.5.427

Ichikawa, N., and Ohira, H. (2004). Eyeblink activity as an index of cognitive processing: temporal distribution of eyeblinks as an indicator of expectancy in semantic priming. Percept. Mot. Skills 98, 131-140. doi: 10.2466/pms.98.1.131-140

Image Analyst (2021). Fit Multiple Gaussians. Available online at: https://www. mathworks.com/matlabcentral/fileexchange/74408-fit-multiple-gaussians

Jung, I., and Kim, J.-S. (2019). Abnormal eye movements in parkinsonism and movement disorders. J. Mov. Disord. 12, 1-13. doi: 10.14802/jmd. 18034

Karson, C. N. (1983). Spontaneous eye-blink rates and dopaminergic systems. Brain 106, 643-653. doi: 10.1093/brain/106.3.643

Kleifges, K., Bigdely-Shamlo, N., Kerick, S.,and Robbins, K.A. (2017). BLINKER: Automated extraction of ocular indices from EEG enabling large-scale analysis. Front. Neurosci. 11:12. doi: 10.3389/fnins.2017.00012

Korosec, M., Zidar, I., Reits, D., Evinger, C., and Vanderwerf, F. (2006). Eyelid movements during blinking in patients with Parkinson's disease. Mov. Disord. 21, 1248-1251. doi: 10.1002/mds.20930 
Kwon, K. A., Shipley, R. J., Edirisinghe, M., Ezra, D. G., Rose, G., Best, S. M., et al. (2013). High-speed camera characterization of voluntary eye blinking kinematics. J. R. Soc. Interface 10:20130227. doi: 10.1098/rsif.2013.0227

Luce RC. Response Times: Their Role in Inferring Elementary Mental Organization. New York, NY: Oxford (1986).

Maffei, A., and Angrilli, A. (2018). Spontaneous eye blink rate: an index of dopaminergic component of sustained attention and fatigue. Int. J. Psychophysiol. 123, 58-63. doi: 10.1016/j.ijpsycho.2017.11.009

Morillon, B., and Baillet, S. (2017). Motor origin of temporal predictions in auditory attention. Proc. Natl. Acad. Sci. U.S.A. 114, E8913-E8921. doi: 10.1073/pnas.1705373114

Nakano, T., Kato, M., Morito, Y., Itoi, S., and Kitazawa S. (2013). Blink-related momentary activation of the default mode network while viewing videos. Proc. Natl. Acad. Sci. U.S.A. 110, 702-706. doi: 10.1073/pnas.1214804110

Nakano, T., and Miyazaki, Y. (2019). Blink synchronization is an indicator of interest while viewing videos. Int. J. Psychophysiol. 135, 1-11. doi: 10.1016/j.ijpsycho.2018.10.012

Sescousse, G., Ligneul, R., van Holst, R. J., Janssen, L. K., de Boer, F., Janssen, M., et al. (2018). Spontaneous eye blink rate and dopamine synthesis capacity: preliminary evidence for an absence of positive correlation. Eur. J. Neurosci. 47, 1081-1086. doi: 10.1111/ejn.13895

Shallice, T. (1982). Specific impairments of planning. Phil. Trans. Royal Soc. London. B, Biol. Sci. 298, 199-209. doi: 10.1098/rstb.1982.0082

Stinear, C. M., and Byblow, W. D. (2003). Role of Intracortical inhibition in selective hand muscle activation. J. Neurophysiol. 89, 2014-2020. doi: 10.1152/jn.00925.2002

Sun, W. S, Baker, R. S., Chuke, J. C., Rouholiman, B. R., Hasan, S. A., Gaza, W., et al. (1997). Age-related changes in human blinks. Passive and active changes in eyelid kinematics. Invest. Ophtalmol. Vis. Sci. 38, 92-99.

Tavano, A., Schröger, E., and Kotz, S. A. (2019). Beta power encodes contextual estimates of temporal event probability in the human brain. PLoS ONE 14:e0222420. doi: 10.1371/journal.pone.0222420

te Woerd, E. S., Oostenveld, R., Bloemde, B. R., Lange, F. P., and Praamstra, P. (2015). Effects of rhythmic stimulus presentation on oscillatory brain activity: the physiology of cueing in Parkinson's disease. Neuroimage Clin. 9, 300-309. doi: 10.1016/j.nicl.2015.08.018

Terao, Y., Fukuda, H., Yugeta, A., Hikosaka, O., Nomura, Y., Segawa, M., et al. (2011). Initiation and inhibitory control of saccades with the progression of Parkinson's disease? Changes in three major drives converging on the superior colliculus. Neuropsychologia 49, 1794-1806. doi: 10.1016/j.neuropsychologia.2011.03.002
Toivanen, M., Pettersson, K., and Lukander, K. (2015). A probabilistic real-time algorithm for detecting blinks, saccades, and fixations from EOG data. J. Eye Mov. Res. 8, 1-14. doi: 10.16910/jemr.8.2.1

Tombaugh, T. N. (2004). Trail making test A and B: normative data stratified by age and education. Arch. Clin. Neuropsychol. 19, 203-214. doi: 10.1016/S0887-6177(03)0 0039-8

Tombaugh, T. N., and McIntyre, N. J. (1992). The minimental state examination: a comprehensive review. J. Am. Geriatr. Soc. 40, 922-935. doi: 10.1111/j.1532-5415.1992.tb 01992

Van Opstal, F., De Loof, E., Verguts, T., and Cleeremans, A. (2016). Spontaneous eyeblinks during breaking continuous flash suppression are associated with increased detection times. J. Vis. 16, 1-10. doi: 10.1167/16.14.21

Wascher, E., Heppner, H., Möckel, T., Kobald, S. O., and Getzmann, S. (2015). Eye-blinks in choice response tasks uncover hidden aspects of information processing. EXCLI J. 14, 207-1218. doi: 10.17179/excli2015-696

Wechsler, D. (1997). Wechsler Adult Intelligence Scale. San Antonio, TX: The Psychological Corporation.

White, O. B., Saint-Cyr, J. A., Tomlinson R. D., and Sharpe, J. A. (1983). Ocular motor deficits in Parkinson's disease: II. control of the saccadic and smooth pursuit systems. Brain 106, 571-587. doi: 10.1093/brain/ 106.3.571

Conflict of Interest: The authors declare that the research was conducted in the absence of any commercial or financial relationships that could be construed as a potential conflict of interest.

Publisher's Note: All claims expressed in this article are solely those of the authors and do not necessarily represent those of their affiliated organizations, or those of the publisher, the editors and the reviewers. Any product that may be evaluated in this article, or claim that may be made by its manufacturer, is not guaranteed or endorsed by the publisher.

Copyright () 2022 Tavano and Kotz. This is an open-access article distributed under the terms of the Creative Commons Attribution License (CC BY). The use, distribution or reproduction in other forums is permitted, provided the original author(s) and the copyright owner(s) are credited and that the original publication in this journal is cited, in accordance with accepted academic practice. No use, distribution or reproduction is permitted which does not comply with these terms. 